\title{
Il Cuore censurato: Edmondo De Amicis e la formazione dello "spirito nazionale" in Argentina
}

\author{
Giovanni Albertocchi \\ Universitat de Girona \\ giovanni.albertocchi@udg.edu
}

\section{Riassunto}

Nel 1884 Edmondo De Amicis si reca in Argentina invitato dal direttore del quotidiano El Nacional a tenere delle conferenze su Garibaldi, Cavour, Mazzini ed altri personaggi della storia italiana. In questo modo prepara anche il terreno per il libro Cuore che arriverà in Argentina tre anni dopo e che sarà accolto trionfalmente, venendo addirittura adottato come libro di testo nella scuola. Verso la fine del secolo però una recrudescenza della cultura nazionalistica che si vedeva minacciata da quella degli emigranti, soprattutto italiani, fa sì che il libro di De Amicis venga proibito dal Consejo Nacional de Educación e sostituito da traduzioni che manipolavano l'originale, nazionalizzandolo in modo da salvaguardare lo "spirito nazionale argentino". L'articolo analizza la tipologia di tre diversi modelli di traduzione: Corazón argentino. Diario de un niño di Carlota Garrido de la Peńa, del 1913; Corazón, Traducción y adaptación para el niño argentino di Germán Berdiales e Fernando Tognetti, del 1937; e Corazón. Adaptación escénica al ambiente nacional del libro de E. de [sic] Amicis, di Germán Berdiales e Pedro Inchauspe, del 1921.

Parole chiave: emigrazione; spirito nazionale; patriottismo, censura, manipolazione.

Abstract. The Cuore censored: Edmondo De Amicis and the formation of the "national spirit" in Argentina

In 1884 Edmondo De Amicis travels to Argentina as a guest of the daily newspaper El Nacional, for lectures on Garibaldi, Cavour, Mazzini and other prominent figures in Italian history. He prepares in this way the arrival of Cuore three years later, which will be a great success: the book will become a textbook in Argentinian schools. Towards the turn of the century, however, a revival of a nationalistic culture which saw as a threat the culture of the new, mainly Italian, immigration, leads to a ban by the Consejo Nacional de Educación on De Amicis' book, which well be replaced by translations manipulating its narrative in order to "nationalize" it according to the "argentinian national spirit". This paper examines three different models of adaptative translation: Carlota Garrido de la Peña, Corazón argentino. Diario de un niño, 1913; Corazón. Traducción y adaptación para el niño argentino, by Germán Berdiales and Fernando Tognetti, published in 1937, and Corazón. Adaptación escénica al ambiente nacional del libro de E. de [sic] Amicis, by Germán Berdiales and Pedro Inchauspe, from 1921.

Keywords: emigration; national spirit; patriotism; censorship; manipulation. 


\section{De Amicis in Argentina ${ }^{1}$}

Nella primavera del 1884 Edmondo De Amicis si reca in Argentina, invitato dal direttore del quotidiano El Nacional di Buenos Aires per una serie di conferenze sui grandi personaggi del Risorgimento, Vittorio Emanuele, Mazzini, Cavour e Garibaldi (Solari, 1946, pp. 15-22).

Benché non avesse ancora pubblicato il libro $\mathrm{Cuore}^{2}$ che gli procurerà una fama immensa anche in Argentina dove poteva contare fra i possibili lettori anche molti connazionali, lo scrittore era già molto noto in questo paese grazie alle traduzioni dei libri di viaggio realizzate in Spagna, ma accessibili naturalmente anche nell'America latina, portate a termine da colui che si può ritenere il suo traduttore ufficiale allo spagnolo, Hermenegildo Giner de los Ríos. ${ }^{3}$

Durante il suo soggiorno lo scrittore ha anche modo di visitare la provincia di Santa Fe (Solari, 1946, pp. 23-27), ai confini dell'immensa pianura della pampa, dove si erano insediate diverse colonie di migranti che formavano "una piccola Italia posta alle frontiere della barbarie". Nell'articolo I nostri contadini in America, apparso nel $1886,{ }^{4}$ De Amicis descrive la vita degli emigranti e le conversazioni avute con loro. In queste, oltre alla soddisfazione per uno status che in Italia era impossibile ottenere, gli emigranti denunciano una progressiva diffidenza manifestata nei loro confronti dal popolo argentino, dovuta probabilmente, annota lo scrittore, al "vago timore d'essere soverchiato dalla popolazione immigrante [che] gli fa sentire spesso il bisogno di rimettere al posto, con una parola altera, i suoi ospiti [...]” (De Amicis, 2016, p. 109). ${ }^{5}$ La diffidenza di cui si parla è già, in nuce, un'avvisaglia delle ostilità che cominciano a manifestare, su scala nazionale, politici ed intellettuali allarmati dal pericolo di essere 'soverchiati' dalla "popolazione immigrante". Negli anni Ottanta del secolo XIX l'esodo migratorio dall'Italia aveva raggiunto in effetti cifre imponenti. Secondo dati statistici elaborati dalla "Dirección de Inmigración", gli

1. Un ringraziamento particolare ad Álex Pérez Vidal che mi ha aiutato a reperire alcuni dei testi utilizzati e a Giuseppe M. Messina che mi ha fornito importanti informazioni sull'emigrazione italiana in Argentina.

2. Edito da Treves, Milano 1886.

3. Giner de los Ríos che, com'è noto, collaborava al progetto pedagogico progressista della Institución libre de Enseñanza, tradusse molte opere di De Amicis, tra cui, oltre naturalmente a Cuore, diversi libri di viaggio come Holanda (Madrid: Librería de V. Suárez, 1883), Costantinopla (Madrid: Librería de V. Suárez, 1883), España (Madrid: Imprenta de Aurelio J. Alaria, 1884). Si veda sull'argomento: García Aguilar, 2006.

4. Larticolo apparve sul Fanfulla della domenica il 24 gennaio 1886. Successivamente, in una versione più lunga fu inserito nel volumetto In America (Ed. Voghera, Roma 1897).

5. Le colonie si rivelarono un'arma a doppio taglio, soprattutto quando si diffuse sulla stampa argentina un articolo dell'economista Girolamo Boccardo in cui sosteneva che la 'colonia' era una sorta di enclave "que conservaba relaciones de amistad y sujeción" con la madrepatria (Bertoni, 2001, p. 31). Boccardo, sia detto per inciso, prese parte, nel 1860, alla controversia insorta tra Manzoni e l'editore Le Monnier sulla proprietà letteraria de I promessi sposi, prendendo le parti dell'editore fiorentino. Lo scrittore gli rispose pubblicando la Lettera al signor professore Girolamo Boccardo intorno ad una questione di cosi detta proprietà letteraria. 
immigrati italiani dal 1857 al 1910 erano circa due milioni; nel decennio 1880-1890 l'incremento era di una media annuale di 64.000 (Rapoport, 2020, pp. 40-41). Lo storico bonaerense Fernando J. Devoto conferma che

pochi decenni della storia argentina furono più 'italiani' della decade del 1880. Lo attestano il peso dei numeri, l'importanza degli italiani nell'economia urbana e rurale, la forza delle loro istituzioni, il prestigio di molti dei loro professionisti e intellettuali, l'influenza dei periodici e persino delle iniziative in campo educativo. Ma nonostante tutto questo o proprio per reazione a tutto questo, pochi decenni in Argentina furono percorsi da un sentimento antitaliano ugualmente forte (Devoto, 2007, pp. 145-146). ${ }^{6}$

La massiccia presenza di immigrati viene considerata in effetti un ostacolo alla formazione del cosiddetto 'spirito nazionale' che rischia di smarrirsi nell'alluvione cosmopolita dei nuovi arrivati. Il deputato Estanislao Zeballos ${ }^{7}$ avverte, in una seduta parlamentare, del rischio per l'Argentina di trasformarsi in una "nación que no tendrá lengua, ni tradiciones, ni carácter, ni bandera" (Bertoni, 2001, p. 21). A preoccupare non era soltanto il loro numero ma anche il fatto che provenissero, come nel caso degli italiani, da paesi in cui si stava consolidando un'idea di nazione che rischiavano quindi di esportare anche all'estero per le proprie velleità patriottiche. Lilia A. Bertoni cita il caso di politici italiani, i quali sostenevano che "la jurisdicción metropolitana debía extenderse allí donde había colonias de connacionales, y donde consecuentemente se prolongaba la nacionalidad italiana" (Bertoni, 2001, p. 17). ${ }^{8}$

\section{Cuore e la "formazione patriottica" argentina}

È presa di mira soprattutto la scuola, in quanto trascurava, si sosteneva da più parti, la "formazione patriottica" degli alunni con contenuti e tematiche che esulavano dalla storia e dall'identità nazionale argentina. Negli anni compresi tra il 1908 ed il 1913, José María Ramos Mejía, presidente del Consejo Nacional de Educación (CNE)

si rese conto, visitando le scuole pubbliche, che in molte c'erano docenti stranieri che non facevano lezione in spagnolo, in alcune erano affissi alle pareti

6. Sempre nella monografia di Fernando J. Devoto leggiamo che secondo il primo censimento nazionale del 1869 gli italiani erano il 3,8 \% della popolazione dell'intero paese che ammontava a 1.877 .490 abitanti (Devoto, 2007, p. 53).

7. Estanislao Zeballos, era, guarda caso, amico di De Amicis: si erano conosciuti durante il viaggio dello scrittore in Argentina. Nel racconto Dagli Appennini alle Ande, c'è un personaggio di cui si dice solo il cognome, Zeballos, «un signore alto con la barba grigia» che aiuta anche economicamente il piccolo Marco nella sua peregrinazione alla ricerca della madre. Secondo Luigi Cepparrone si tratta del deputato Estanislao Zeballos e «l'episodio vuole essere un atto di omaggio nei confronti [dell'] argentino [...], del quale De Amicis era diventato amico, durante il suo viaggio» (Cepparrone, 2012, p. 151).

8. Nel 1887 una circolare del governo di Francesco Crispi "raccomandava ai consoli di fare tutto il possibile per stimolare la celebrazione delle feste nazionali italiane [...] per mantenere vivo il patriottismo tra gli emigranti” (Devoto, 2007, p. 152). 
delle aule ritratti di Umberto I, della regina Margherita e di altri illustri italiani, donati dai genitori degli alunni, in numerose altre si usava come testo patriottico Cuore di Edmondo De Amicis, e non i libri che esaltavano le gesta degli argentini [...] (Devoto, 2007, p. 316).

Il libro era arrivato in Argentina nella traduzione eseguita nel 1887, a pochi mesi di distanza dall'edizione italiana, da Hermenegildo Giner de los Ríos, ottenendo subito un enorme successo 9 e, come documentano Fernando Degiovanni (2007) e Liliana H. Zuntini (2011), a partire dal 1894 viene adottato come libro di testo nella scuola elementare. ${ }^{10}$ Con il risultato che proprio "la institución pensada para operar como eje del programa de nacionalización cultural había caído presa del enemigo al que quería combatir [...]" (Degiovanni, 2007, p. 104).

E assai noto il divertente episodio riferito dal già citato José M. Ramos Mejía nella relazione su La educación común en la República Argentina, in cui esponeva i risultati delle sue ispezioni in molte scuole del paese:

La mayoría de los libros de texto usados en las Escuelas eran extranjeros, ofreciendo los inconvenientes que es de suponer. Uno de los más difundidos era "Cuore" de D'Amicis [sic], obra maestra de este distinguido escritor y destinada a ensalzar las glorias de Italia, a contribuir a su unidad y a despertar el amor al Rey. Para que pueda explicarse la influencia de estas obras en el espíritu de los nińos, referiré la siguiente anécdota: un distinguido médico que no tengo inconveniente en nombrar, el Dr. Rafael Herrera Vega, escuchaba en cierta ocasión a un niño que recitaba un trozo de D’Amicis sobre la bandera. Como en él se hiciera alusión a la bandera tricolor de la patria, dirigiéndose al niño le preguntó: “¿Y cuál es esa bandera tricolor?”. "La bandera de mi patria”, contestó el niño sin vacilar (Ramos Mejía, 1913, p. 9).

La pericolosità del libro è denunciata, senza mezzi termini, da un illustre pedagogista, Juan P. Ramos, secondo il quale, si legge in Valeria Sardi, "la novela de De Amicis debe ser desterrada del circuito escolar porque produce efectos

9. Seguirono riedizioni argentine, sempre nella traduzione di Giner de los Ríos, come quella pubblicata nella collana Biblioteca La Nación (Buenos Aires 1905). Assai diffusa la versione curata dall'insegnante Emma Bedogni, pubblicata da un editore italiano, Natalio Tommasi, probabilmente, secondo Fernando Degiovanni, prima del 1914, anno della morte dello stesso Tommasi. "Es probable que esta edición económica de Corazón haya sido uno de los principales motivos de preocupación para quienes veían en la lectura de la obra un elemento contraproducente para la asimilación de los inmigrantes y la consolidación del nacionalismo entre los nativos (Degiovanni, 2007, pp. 106-107). A proposito della lettura di Cuore c'è un suggestivo ricordo scolastico del filosofo argentino Mario Bunge: "Nunca nos dijeron cuántos eran los inmigrantes ni por qué habían venido al país. En cambio, en la escuela primaria nos habían hecho leer Corazón, de Edmundo De Amicis, que contaba la conmovedora gesta de un niño italiano que vino huyendo de la miseria. Pero nadie nos contó que De Amicis había sido un militante socialista [...]” (Bunge, 2014, p. 39).

10. Parti del libro circolavano anche in diverse antologie: "Enrique García Velloso incluyó veinte capítulos de Corazón en su obra Prosa selecta editada por Estrada [1905] y Andrés Ferreyra - el autor del Nene - adaptó doce capítulos para incluirlos en su libro de lectura Aventuras de un niño de la misma editorial [1898]" (Spregelburd, 2018, p. 236). 
negativos en el lector, les hace sentir 'sentimientos extraños a sus idiosincrasias nacionales' y obstaculiza la formación del sentimiento argentino necesario para combatir el cosmopolitismo y producir la consciencia nacional" (Sardi, 2011, p. 70). Per non parlare poi dei figli degli emigranti italiani per i quali la lettura del libro minacciava di rallentarne o addirittura di comprometterne l'integrazione nella cultura della nuova patria. ${ }^{11}$ In quegli anni era divampata inoltre la polemica contro le scuole italiane accusate di essere emissarie di una forma di imperialismo culturale "capaz de influir sobre el elemento local y hasta predominar en él" (Bertoni, 2001, p. 25). L'ex presidente della Repubblica, Sarmiento, fu uno dei più accesi avversari delle scuole italiane, con una serie di articoli apparsi nel gennaio del 1881 sul quotidiano El Nacional dai titoli di per sé molto eloquenti: Las colonias sin patria; Las escuelas italianas: Su inutilidad; Una Italia en América; La nostalgia en América, ecc. ${ }^{12}$ In un articolo pubblicato sempre su El Nacional, nel 1882, esprimeva degli assurdi timori sulle mire espansionistiche dell'Italia: "esto lo han hecho otras veces los ingleses para apoderarse sin título de las islas Falkland, ¿por qué no lo haría la Italia?” (Bertoni, 2001, p. 26).

Di questo clima di recrudescenza nazionalista fa le spese anche il libro di De Amicis che alla fine viene bandito dalla scuola. Si convoca addirittura un concorso per rimpiazzarlo con un'altra lettura per ragazzi ma con contenuti argentini. Ricardo Rojas, uno dei più acerrimi avversari del 'cosmopolitismo', in una relazione del 1909, intitolata La restauración nacionalista: informe sobre educación, afferma compiaciuto che "El Consejo [Nacional de Educación], como era lógico, ha mandado suprimir aquellos cuadros [i ritratti citati poc'anzi] y el uso del Cuore. Lo ha reemplazado, provisionalmente, por los Recuerdos de Provincia ${ }^{13}$ hasta que un concurso pueda proveerlos de otro más didáctico" (Rojas, 2011, p. 245). Comunque la "falta de material didáctico" di cui deve prendere atto e a cui si deve aggiungere la constatazione che Cuore si era rivelato un modello insuperabile di lettura scolastica, accompagnato per di più da un incredibile successo, dà origine ad un curioso fenomeno che la studiosa Valeria Sardi, nel suo documentatissimo saggio, definisce "traducción cultural", consistente nel riutilizzare il libro di De Amicis trapiantandolo in un

11. A proposito dell'importanza di Cuore per la comunità italiana in Argentina, Valeria Sardi dice che: "la novela del escritor turinés venía a reponer un vacío cultural en el exilio forzado de miles de inmigrantes que encontraban en el texto su pertenencia nacional inscripta en la historia de Enrique y que, a través de la lectura, podían mantener un lazo simbólico con sus propios orígenes, con los familiares que habían quedado en la península, con su propia identidad desgarrada" (Sardi, 2011, p. 65).

12. Gli interessi della colonia italiana erano difesi da due giornali, L'operaio Italiano nato nel 1874, e soprattutto da La Patria degli Italiani, fondato nel 1876 da Basilio Cittadini. Nel 1887 La Patria degli Italiani raggiunse una tiratura di 11.000 copie, una diffusione che lo rese il quarto giornale del paese (Deschamps, 2009, p. 320).

13. È l'autobiografia scritta nel 1850 da Domingo Faustino Sarmiento, Presidente della Repubblica dal 1868 al 1874 ed autore di Facundo o civilización y barbarie en las pampas argentinas, uno dei testi canonici della letteratura ispano-americana (Imprenta del Progreso, Santiago de Chile 1845). 
contesto argentino secondo gli opportuni codici culturali della nazione. Dato che il libro è costruito su un duplice progetto, quello moralistico-edificante e quello patriottico, si trattava di mantenere il primo, che rientrava negli obbiettivi di qualsiasi educazione scolastica dell'epoca, e di adattare l'altro alla storia argentina, sostituendo, in poche parole, il Risorgimento italiano con la guerra di indipendenza della ex colonia dalla Spagna. La "traducción cultural - avverte Valeria Sardi - implica una manipulación editorial en tanto se modifica el texto por la aparición de subtítulos, resúmenes de capítulos, adiciones, expansiones, supresiones, abreviaturas, cambios en la estructura como en el estilo del texto [...]" (Sardi, 2011, p. 107). Si tratterebbe in sostanza di una forma di 'plagio' autorizzato in cui conciliare le 'esigenze di mercato' con quelle patriottiche della salvaguardia dello 'spirito nazionale argentino'.

Fra i diversi modelli di "traducción cultural” che presenta Valeria Sardi nel suo saggio, ho scelto di analizzarne tre che rappresentano altrettante tipologie di intervento sul testo originale. Una più radicale, Corazón argentino. Diario de un niño di Carlota Garrido de la Peña, in linea con i dettami censori del $\mathrm{CNE}^{14}$ che in effetti la premierà inserendo il suo libro nel canone delle letture scolastiche. ${ }^{15}$ Poi Corazón. Traducción y adaptación para el niño argentino di Germán Berdiales e Fernando Tognetti, dove si recupera, con le opportune cautele, il Cuore originario, ${ }^{16}$ adattandolo alla "comprensión y sensibilidad de nuestros niños». Berdiales è anche autore, in collaborazione con Pedro Inchauspe di una succinta versione teatrale: Corazón. Adaptación escénica al ambiente nacional del libro de E. de [sic] Amicis.

\section{Un Corazón argentino}

L'autrice, Carlota Garrido de la Peńa, illustra e giustifica la sua operazione con "Dos palabras de advertencia" che rivolge subito al lettore:

Un eminente italiano escribió hace algunos años un libro admirable que ha sido traducido a muchos idiomas y que él dedicó a los niños de su país. El éxito de este libro consistió en que, separándose del espíritu de los textos escolares de lectura, llenos de páginas de ciencia, de industria y de una moral demasiado ficticia, se dirigió directamente al corazón de sus pequeños compatriotas interesando sus sentimientos infantiles con narraciones de la vida real, de cada una de las que habían de sacar una enseñanza. Sin pretender haber escrito un libro como el de D’Amicis [sic] (Corazón), pero, lo confieso, habiendo seguido modestamente su huella, ofrezco hoy a todos los niños y niñas de mi país que ya sepan leer corrientemente, el presente libro de lectura, que siendo las impresiones de un escolar modelo, es al mismo tiempo bien intencionado y nacional. Lo ofrezco a mis colegas los maestros de escuela,

14. De Amicis non figura in copertina quale autore, ma soltanto nel prologo.

15. Il libro di Garrido de la Peña si colloca "a la vanguardia en una guerra en la que los oponentes se veían obligados a valerse de las armas del enemigo para triunfar sobre él” (Degiovanni, 2007, p. 109).

16. Il nome di "Edmundo De Amicis" è ben visibile nella copertina. 
y quedaré recompensada si los niños hallan placer en su lectura y tratan de imitar a alguno de sus protagonistas más simpáticos, y si los maestros utilizan la moral de sus páginas, pues pienso que la misión más alta y excelente que puede realizar un educador que quiera servir a su país, es orientar hacia el bien el pensamiento y el corazón de los ciudadanos y de las madres del porvenir (Garrido de la Peńa, 1913, pp. 5-6). ${ }^{17}$

La premessa è ricca di temi che Garrido de la Peña svolgerà diligentemente nel libro. Si parte da una debole traccia ("huella") deamicisiana per imbastire una storia sostanzialmente diversa, redatta, anche se non in forma di diario come assicura il titolo, da un alunno modello e soprattutto "bien intencionado y nacional". ${ }^{18}$ C'è poi un invito ai colleghi (Carlota era maestra) a usare Corazón argentino come un libro di testo. ${ }^{19}$ In effetti molti capitoli, soprattutto quelli che hanno come tema la storia nazionale, ma anche quelli di contenuto edificante o puramente scolastico, si concludono con una "Nota a los Señores Maestros" del tipo: "Hágase comentar cada una de las páginas históricas", "Hágase hacer biografías cortas de los argentinos ilustres aprovechando los datos históricos de estas páginas", "Resumen y explicación sobre esta página patriótica. Hágase hacer una composición sobre la patria”, 20 "Aprovéchese el tema de la lección para afear a los niños el vicio del cigarro" e, dulcis in fundo, anche un'esortazione corporativa, "Aprovéchese el tema para despertar en los alumnos la estimación y afectos por el Maestro". A dire il vero, stando al racconto di Ángel Revilla, controfigura di Enrico Bottini, non ce ne sarebbe bisogno: a parte il cattivo di turno che qui si chiama Andrés Cheni che però non verrà espulso come Franti ma si redimerà, tra il maestro ed i suoi alunni c'è un vero e proprio idillio, fomentato dai due 'leader', Juancito González (Derossi) e Pardo (Garrone). All'appello dei buoni sentimenti non manca neppure la versione argentina del ragazzo calabrese che qui è un indio, Quilito, che "también es argentino - precisa il maestro - ejemplar más puro de una raza noble, casi desaparecida", ${ }^{21}$ e il figlio di emigranti, Vioncarlo, "un italianito hijo de colonos [dal] corazón noble y caritativo" come dimostra compiendo un'azione eroica (vedi il 'collega' Robetti) per cui gli verrà conferita nel "Palacio Escolar" di Buenos Aires una medaglia d'oro mentre la banda esegue, in suo onore, la "marcha de Garibaldi".

17. Nel libro Mis recuerdos, l'autrice dice di aver finanziato la prima edizione del libro con i suoi risparmi: "Había jugado a una carta todo un ańo de mis sueldos de maestra" (Garrido de la Peña, 1935, p. 36).

18. La storia si svolge in una scuola elementare di Santa Fe. L'autrice aveva iniziato in questa città la sua attività di docente.

19. Afferma l'autrice in Mis recuerdos: "Sujeté a los programas de lectura, historia y moral cívica existentes los lineamentos y la médula del texto» (Garrido de la Peña, 1935, p. 28).

20. Una circolare del CNE prescriveva ai maestri di fomentare l'abitudine di far cantare agli alunni l'inno nazionale e di far trattare "temas históricos en composiciones orales y escritas" (Bertoni, 2001, p. 117).

21. "Grazie" anche alla "guerra" o "conquista del desierto" (1878-1885) promossa dal governo argentino, che nei decenni precedenti aveva sterminato le popolazioni indigene. 
Le "Dos palabras de advertencia" si chiudono citando fra i destinatari del libro "las madres del porvenir". Non è questo l'unico accenno 'di genere': in Corazón argentino la presenza femminile, trattandosi di una scrittrice, è assai più consistente che nell'archetipo italiano, grazie soprattutto alla sorella del protagonista, Adela, che si prodiga in attività benefiche che vanno ad incrementare la quota dei buoni sentimenti e soprattutto il suo ruolo all'interno della storia. Invita a pranzo le compagne meno fortunate (El banquete de Adela), dando vita a quella che la maestra definisce "una reunión de ángeles"; organizza una gita in un orfanatrofio, mandando in visibilio i piccoli ospiti con un cestello di leccornie (Una visita al asilo); protegge una compagna afflitta da cifosi (La jorobadita), imitando l'esempio del nostro Garrone che ventisette anni prima si era occupato con affetto analogo del "povero gobbino" Nelli. Nelle edizioni successive di Corazón argentino, il ruolo di Adela diventa, in un certo senso, militante: in un componimento che viene trascritto dal fratello, intitolato Damas argentinas, la fanciulla rivendica il ruolo avuto dalle donne nella conquista dell'indipendenza, affiancandole così agli "Argentinos illustres", a cui, come si vedrà più avanti, sono dedicati diversi capitoli del libro. Le donne, afferma con orgoglio la Garrido, sono presenti anche "en muchas instituciones sociales y humanitarias que prueban el temple de su carácter y de su corazón", come dimostrano le loro attività nel "Consejo Nacional de Mujeres de la República Argentina” (Garrido de la Peña, 1921, pp. 329-331).

Come si è visto i ragazzi della "Sezione Baretti" ci sono quasi tutti, rivisti secondo il patto con il lettore stabilito nella premessa. Del libro di De Amicis sopravvive qualche residuo di episodi edificanti come quello del bambino cieco che suscita la compassione dei compagni di Ángel (Enrico), episodio che comprime, utilizzando soltanto il registro emotivo, il capitolo di Cuore, I ragazzi ciechi, ove si narra la visita della classe all'Istituto che li accoglie e li 'rieduca', dando loro la possibilità di una certa autonomia. De Amicis dimostrava così quanto confidasse nei benefici che può offrire il progresso sociale e scientifico. ${ }^{22}$ Un trattamento analogo riserva Garrido de la Peña all'episodio della "sordomuda", che è la riduzione, in chiave patetica, del capitolo La sordomuta, in cui Enrico Bottini ha sì modo di commuoversi, ma anche di fare una visita istruttiva all'istituto che ospita Gigia, la figlia del giardiniere, e di osservare le tecniche, per quell'epoca avanguardistiche, con cui si insegna ai sordomuti a parlare ed a comunicare con quella che oggi si chiamerebbe la lingua dei segni (De Amicis, 2012, pp. 249-257).

Nel Corazón argentino la storia italiana è sostituita, come si è già detto, da quella locale che comunque coincide, più o meno, sia come cronologia che come obbiettivi (l'indipendenza, la fondazione dello stato ed il patriottismo), con quella italiana. I temi storici, destinati a "fomentar en esas tiernas almas el santo fuego del patriotismo" (Garrido de la Peña, 1913, p. 20), obbiettivo, tra l'altro, che anche De Amicis avrebbe potuto sottoscrivere, sono distribuiti

22. Vedi a questo proposito l'importanza dell'educazione fisica nel racconto Amore e ginnastica (De Amicis, 1892, pp. 59-118). 
nelle diverse attività scolastiche (lezioni, gite ai sacrari della patria, temi svolti dagli alunni, lettere varie indirizzate al protagonista ed inserite nel testo, ecc.), dove in modi diversi si rievocano gli "Argentinos ilustres": José de San Martín, Manuel Belgrano, Bernardino Rivadavia, Domingo F. Sarmiento, ecc. Al patrimonio storico patriottico appartengono anche i due piccoli eroi dei racconti mensili che il maestro detta agli alunni: il Soldado de la patria (adattamento de La piccola vedetta lombarda) e Tormenta, la storia di un ragazzo di colore, così soprannominato per la sua vivacità, che si conquista i galloni come trombettiere di un reparto che combatte nella guerra contro il Paraguay. I giovinetti protagonisti degli altri racconti mensili ("Cuentos mensuales"), la cui trama ha poco a che vedere con quelli di De Amicis, sono però, come i compagni italiani, modelli di eroismo e di abnegazione. Fra questi citerei El grumete ('Il mozzo'), un emigrante dalla Galizia (un "galleguito") che diventa guardiamarina e che esibisce davanti al padre la sua riconoscenza verso la patria di adozione con l'apologia dell'integrazione come prescriveva certa ideologia dell'epoca: ${ }^{23}$

La amo [l'Argentina] porque me ha admitido entre sus hijos, me ha brindado los beneficios de la educación al darme una carrera honrosa [...] y te aseguro que en cualquier tiempo derramaré gustoso por defenderla la última gota de mi sangre (Garrido de la Peńa, 1913, p. 112).

Il libro che, come già detto (cfr. n. 16) Garrido de la Peńa pubblica a proprie spese, ottiene il gradimento del Consejo de Educación Nacional che lo inserisce nella lista dei libri di testo consigliati. A ciò si aggiunge un grande successo di vendite anche al di fuori del mercato scolastico, che le varrà un contratto con una casa editrice di Buenos Aires, la Cabaut \& Cía., che pubblicherà nel giro di una decina di anni ben sei edizioni del libro (Maristany, 2001, p. 439).

\section{Corazón. Traducción y Adaptación para los niños argentinos}

Gli autori, Germán Berdiales e Fernando Tognetti, recuperano, come si è detto, il libro ed il nome di De Amicis che riappare nella copertina. Nel prologo, intitolato "Bienvenida", Rafael Alberto Arrieta illustra il senso della "adaptación" di un testo definito ammirevole, il cui fascino presso il pubblico infantile argentino era però ostacolato da elementi di "sorda opacidad para la comprensión y la sensibilidad de nuestros niños". Seguono gli ambiti su cui si è dovuto intervenire per rimuoverne gli ostacoli: "Nombres, próceres, modalidades, hábitos correspondientes a un país extranjero" con il vantaggio che la "feliz adecuación 'nacionaliza', definitivamente, la obra transvasada". Arrieta escogita una sequenza assai suggestiva per rappresentare l'operazione, suggerendo che sarebbe "como si el propio alunno di terza d'una scuola municipale

23. A proposito dell'integrazione degli immigrati, il deputato Estanislao Zeballos aveva affermato in Parlamento, nel 1887, la necessità di "atraer hacia nuestra patria a todos los habitantes del mundo que quieran vivir en ella e inculcar en el corazón de los extranjeros el sentimiento de nuestra nacionalidad" (Bertoni, 2001, p. 17). 
d'Italia, residente en la Argentina desde su juventud, hubiese adaptado su lejana storia d'un anno scolastico, para sus nietos argentinos" (E. De Amicis, 1937, p. 10). La nuova versione, che Arrieta definisce efficacemente "trasplante", conserva comunque la "frescura, la gracia, la emotividad del relato, así como el dibujo de los caracteres y la trama de los episodios". Valeria Sardi rivela giustamente che il prologo "funciona como sanción de la crítica y de la Academia ya que pertenece a Rafael Alberto Arrieta, profesor de Literatura en el Colegio Nacional de la Universidad Nacional de La Plata y presidente de la Academia Argentina de Letras" (Sardi, 2011, p. 112).

Il libro mantiene la struttura di un diario (che nella versione di Garrido de la Peña era scomparsa) redatto da Enrique, in cui si raccontano le vicende di un anno scolastico in una scuola elementare situata nella Calle Piedra di Buenos Aires. ${ }^{24}$ Tra i compagni di classe di Enrique, alcuni, come Perboni, Derossi, Garrone, Stardi, ecc. rimangono fedeli all'anagrafe deamicisiana, altri, come Coretti, Votini e Carlo Nobis assumono le generalità locali di Correa, Benítez e Carlos Noble. Non mancano poi i casi esotici di Robetti, Nelli e Garoffi che mutano in "Roberts", "O’Nell" [sic] e "Garoff”, probabilmente per assicurare la presenza di altre componenti nazionali dell'immigrazione. Franti diviene inspiegabilmente Franco e non finirà all'ergastolo, come annunciano enfaticamente i suoi compagni italiani, ma nel "Reformatorio de Marcos Paz". Un discorso a parte merita il "ragazzo calabrese" che, nel libro di De Amicis, arriva pochi giorni dopo l'inizio dell'anno scolastico e che viene presentato dal maestro come "un piccolo italiano nato a Reggio di Calabria, a più di cinquecento miglia di qua". Berdiales e Tognetti sono costretti a ricorrere ad un "chico mendocino [...] nacido al pie de los Andes, en Mendoza, a más de mil kilómetros de aquí", con risultati piuttosto enfatici in quanto non suffragati, come in Cuore, dall'apporto emotivo dell'unificazione nazionale. Ai "próceres" ('padri della patria') di cui si parla nel prologo, viene riservata una "feliz adecuación" al contesto argentino, cercando però di mantenere la massima fedeltà (un autentico lavoro da certosino) ai testi in cui sono evocati, siano essi una lezione del maestro, un componimento scolastico o un intervento edificante del padre di Enrico. Così, ad esempio, la commemorazione del generale Manuel Belgrano "nacionaliza" quella di Vittorio Emanuele; ${ }^{25}$ lo stesso accade con José de San Martín e Giuseppe Garibaldi o con Domingo F. Sarmiento ed il conte di Cavour. Il rientro a Torino di Umberto di Savoia (Re Umberto) è sostituito da quello a Buenos Aires del Presidente Roque Saenz Peña (El Presidente Saenz Peña). ${ }^{26}$ Rimane, invece, intatto l'omaggio a 'José'

24. I toponimi sono di conseguenza aggiornati secondo la nuova ambientazione bonaerense. La Plaza de Mayo fa la parte del leone, 'traducendo' diversi luoghi torinesi, come Piazza San Carlo, il Corso e Piazza Castello.

25. In entrambi i casi la commemorazione è affidata al 'primo della classe', l'impareggiabile Derossi.

26. Il padre di Correa (Coretti) che aveva combattuto nello stesso reggimento del personaggio, viene da questi riconosciuto. Ha l'orgoglio di stringergli la mano con cui accarezzerà poi il figlio, avvertendolo, solennemente, in Cuore come in Corazón, che "Questa è una carezza 
Mazzini, titolare, come in Cuore, oltre che di innumerevoli benemerenze anche di uno straordinario affetto per la madre: "adoraba a su madre", è la frase che ne fa testimonianza, a cui però Berdiales e Tognetti aggiungono con orgoglio un tocco 'nostrano': "como entre nosotros adoró a la suya Sarmiento".

Il processo di nazionalizzazione continua con il questionario patriottico proposto dal Signor Bottini al figlio: "Perché amo l'Italia?", trasformato in “¿Por qué amo a la República Argentina?”. L'amor di patria troverà la sublimazione nelle ultime pagine del diario, con il panegirico, sempre a cura del padre di Enrico-Enrique, ${ }^{27}$ intitolato rispettivamente Italia e Argentina. Sia in Cuore che in Corazón il patriottismo investe anche la geografia della patria evocata in entrambi i testi con i nomi delle città più illustri. La geografia, naturalmente 'adattata', presiede, come nel libro di De Amicis, la ripartizione regionale dei modelli di eroismo proposti nei racconti mensili. ${ }^{28}$ Eccone la lista: El pequeño patriota puntano (Il piccolo patriotta padovano), El pequeño copista rosarino (Il piccolo scrivano fiorentino), El pequeño observador tucumano (La piccola vedetta lombarda), El enfermero del "tata" (L'infermiere di Tata), El tamborcito salteño (Il tamburino sardo), Sangre correntina (Sangue romagnolo). Nei due racconti di argomento patriottico, l'ambientazione risorgimentale cede il posto, come vuole la prassi, alla guerra di indipendenza contro la Spagna. Valor civil (Valore civile) si svolge, per così dire, in sede, a Buenos Aires come a Torino, e racconta l'assegnazione di una medaglia al valor civile al ragazzo che salvò un compagno dall'annegamento. Un caso a parte è De los Apeninos a los Andes (Dagli Appennini alle Ande), racconto che era tra l'altro già apparso in diverse antologie scolastiche argentine. ${ }^{29}$ L'originale, data l'ambientazione 'autoctona', viene naturalmente rispettato: si riscontra però una suggestiva modifica dei verbi che indicano la rotta seguita dal protagonista, di cui si dice che "vino de Génova a América" anziché "andò da Genova in America", con cui i traduttori intendono aggiornare il nuovo punto di vista.

\section{Corazón, una adaptación escénica}

Germán Berdiales è anche autore, in collaborazione con Pedro Inchauspe, di Corazón - "Adaptación escénica al ambiente nacional del libro de E. de [sic] Amicis" - pubblicata nel 1921 sul Monitor de la Educación Común (EMEC), rivista del "Consejo Nacional de Educación" creata nel 1881 da Domingo A. Sarmiento. ${ }^{30}$ Anche qui la premessa serve da protocollo con cui giustificare

del re", "Esta es una caricia del presidente".

27. «En la familia Bottini - sostiene José J. Maristany - se habla poco pero se escribe mucho» (Maristany, 2001, p. 439).

28. A parte, com'è evidente, Naufragio.

29. Oltre alla già citata Prosa selecta (1905) di Enrique García Velloso, anche in Lectura selecta (1922).

30. L'adattamento verrà inserito, successivamente, in una antologia, sempre curata da G. Berdiales, in collaborazione con P. Inchauspe, intitolata La fiesta de mi escuelita. Para la escena y para el aula. (Comedias - Diálogos - Monólogos y Discursos, Prólogo por Víctor 
l'operazione: "Enamorados del libro de Edmundo De Amicis, hemos querido teatralizarlo, adaptándolo a nuestro ambiente", cercando però di "conservar los valores dramáticos y los caracteres que trazó el autor". Si tratta di quattordici brevi scene, tutte ambientate in una "aula de una escuela porteña" dove si svolgono o meglio si raccontano, visto che mancano gli 'esterni', alcuni episodi salienti del testo originale. Una sorta di puzzle dove i personaggi ripropongono, anche attraverso spezzoni di dialoghi originari, il ruolo interpretato nel libro di De Amicis. Così, mentre si alza il sipario, appare Coreta (Coretti) ${ }^{31}$ che ripassa tenacemente la lezione di grammatica con l'aiuto di Deroso (Il mio compagno Coretti). Poi entrano in scena Garofi che traffica come un rigattiere, con le tasche piene di cianfrusaglie (I miei compagni); Nobis, il superbo; Crosi con "un brazo inmóvil, pegado al cuerpo" che, sbeffeggiato da Franti ${ }^{32}$, lancia contro di lui il calamaio che colpisce inavvertitamente il maestro. Garrón si assume la colpa e riceve, per la sua generosità (Un tratto generoso), le lodi del maestro (“Tienes un alma noble, Garrón”). Enrique perde naturalmente il ruolo di narratore: sulla scena, da regista qual era prima, diviene una semplice comparsa.

Le vicende storiche, impossibili da rappresentare, sono semplicemente 'appese' come illustrazioni patriottiche alle pareti dell'aula ed evocate dai versi della poesia A mi bandera del "poeta soldado" Juan Chassaing che Deroso dovrà recitare l'indomani durante la premiazione degli alunni meritevoli. Il sipario cala sui primi versi "Página eterna de argentina gloria / melancólica imagen de la patria [...]" che Deroso, su invito del maestro, prova a recitare in anteprima ai compagni seduti davanti a lui.

\section{Conclusione}

Come testimoniano Fernanda E. Bravo Herrera (2014, p. 2) e Roberta P. Spregelburg (2018, p. 241) la censura e le manipolazioni del libro dureranno, con risultati alterni legati alla recrudescenza o all'allentamento delle istanze nazionalistiche, fino al 1940. Comunque, a detta di Valeria Sardi, il progetto di nazionalizzazione fallisce, in quanto non riesce a soppiantare "en la memoria de los lectores [...] el hipotexto italiano" (Sardi, 2011, p. 18). Di fatto, nel 1946, riferendosi al lascito culturale ed emotivo di Cuore, Juan Antonio Solari ebbe a scrivere significativamente: "Todos tenemos con E. de Amicis, desde

Mercante), A. Cabaut \& Cía., Buenos Aires 1931, pp. 11-24. L’autore del prologo afferma che "Berdiales ha tenido [...] la feliz idea de adaptar algunas páginas maestras de autores como Víctor Hugo, Oscar Wilde, Ricardo Palma, Ada M. Elflein, Edmundo de Amicis, Clemente Onelli, a la comprensión y sentimentalidad del niño sin que la fábula perdiera el encanto originario" (p. 8).

31. I personaggi recuperano i nomi che erano stati loro assegnati nella prima traduzione spagnola di H. Giner de los Ríos, nel 1887: Garrón, Deroso, Coreta, Enrique, el albañilito, Precusa, Garofi, Estardo, mentre quelli di Nobis e Franti restano uguali.

32. Che qui appare circondato dalla sua classica aureola di malvagità, sottolineata, come in Cuore, dalla famosa invettiva del maestro: "Franti estás matando a tu madre" mentre "el muy infame sonreía" ("Franti, tu uccidi tua madre! [...] E quell'infame sorrise"). 
nuestra infancia, una deuda. A través de las páginas de Corazón nos pusimos en contacto con su noble espíritu y fuimos sus amigos al serlo de los muchachos de la escuela [...] cuyas vidas hemos seguido día a día, con sus alegrías y dolores, sus esperanzas y diabluras infantiles [...]. Son recuerdos imperecederos, emociones puras y hondas" (Solari, 1946, p. 1).

\section{Riferimenti bibliografici}

Berdiales, G., \& Inchauspe P. A. (1921). Corazón. (Adaptación escénica al ambiente nacional del libro de E. de [sic] Amicis), El Monitor de la educación común, 40 (586), 56-64.

Bertone, G. (1983). La patria in piroscafo. In E. De Amicis, Sull'Oceano. Genova/ Ivrea: Herodote.

Bertoni, L. A. (2001). Patriotas, cosmopolitas y nacionalistas. La construcción de la nacionalidad argentina a fines del siglo XIX. Buenos Aires: Fondo de Cultura Económica.

Bravo Herrera, F. E. (2014). De Amicis en Argentina, Claves, XXIII (228).

Bunge, M. (2014). Memoria. Entre dos mundos. Barcelona: Gedisa.

Cepparrone, L. (2012). Gli scritti americani di Edmondo De Amicis. Soveria Mannelli: Rubbettino Editore.

De Amicis, E. (1887). Corazón: diario de un niño, (Traducido al español de la 44a edición italiana por H. Giner de los Ríos. Versión revisada por el autor y exclusivamente autorizada para Espańa y América). Madrid: Librería de los sucesores de Hernando.

De Amicis, E. (1892). Fra scuola e casa. Bozzetti e racconti. Milano: Treves.

De Amicis, E. (1937). Corazón. (Prólogo de R. A. Arrieta; Traducción y Adaptación para el nińo argentino de F. Berdiales \& F. Tognetti). Buenos Aires: Anaconda.

De Amicis, E. (2012). Cuore. (Introduzione di F. Custodi; Prefazione di B. Gambarotta). Milano: Garzanti.

De Amicis, E. (2016). I nostri contadini in America. (Ristampa anastatica dell'edizione Voghera del 1897). Introduzione di Ornella Moroni. Sassari: C. Delfino editore.

Degiovanni, F. (2007). Los textos de la patria. Nacionalismo, politicas culturales y canon en Argentina. Buenos Aires: Beatriz Viterbo Editora.

Deschamps, B. (2009). Echi d'Italia. La stampa dell'emigrazione. In AA.VV., Storia dell'emigrazione italiana, Arrivi. II. Roma: Donzelli, pp. 313-334.

Devoto, F. J. (2007). Storia degli Italiani in Argentina. Roma: Donzelli.

García Aguilar, M. (2006). Traducción y recepción literaria de la obra de Edmondo De Amicis en Espańa (1877-1908). Estudio crítico y repertorio bibliográfico, Sendebar. 17, 99-118.

Garrido de la Peña, C. (1913). Corazón argentino: diario de un niño. Valencia: F. Sempere.

Garrido de la Peña, C. (1921). Corazón argentino: diario de un niño. Buenos Aires: Cabaut \& Cía.

Garrido de la Peña, C. (1935). Mis recuerdos. Rosario: La Cervantina.

Governo della Repubblica Argentina. (1870). Guida per l'emigrante italiano alla Repubblica Argentina. Documento ufficiale. Firenze: A. Bettini Librajo-Editore.

Magnani, I. (2015). I migranti nella letteratura italiana. Dall'assenza all'equivalenza. Zibaldone. Estudios italianos. III (1), 260-270. 
Maristany, J. J. (2001). Un libro para "el corazón de los ciudadanos y las madres del porvenir": "Corazón argentino" de Carlota Garrido de la Peña. In M. C. Vázquez \& S. Pastormerlo, Literatura argentina: perspectivas de fin de siglo. Buenos Aires: Eudeba, pp. 433-446.

Parisi, G. (1907). Storia degli Italiani nell'Argentina. Roma: Voghera.

Ramos Mejía, J.M. (1913). La educación común en la República Argentina, años 1909-1910 (Consejo Nacional de Educación). Buenos Aires: Talleres Gráficos de la Penitenciaría Nacional.

Rapoport, M. (2020). Historia económica, social y politica argentina (1880-2003). Buenos Aires: Planeta.

Rojas, R. (2011). La restauración nacionalista: informe sobre educación. Buenos Aires: Unipe. Editorial Universitaria. Versione online. <http://biblioteca.clacso.edu.ar/ Argentina/unipe/20171121055305/pdf_336.pdf >.

Sardi, V. (2011). Políticas y prácticas de lecturas. El caso "Corazón” de Edmundo De Amicis. Buenos Aires: Miño y Dávila.

Solari, J. A. (1946). E. De Amicis y la Argentina. Buenos Aires: Sociedad Luz.

Spregelburd, R. P. (2018). De los Apeninos a los Andes: Las lecturas de Corazón en la escuela argentina. In AA.VV. Para una historia de la enseñanza de la lectura y escritura en Argentina. Buenos Aires: Miño y Dávila, pp. 229-251.

Zuntini, L.H. (2011). Edmundo De Amicis. Con los “ojos de la mente”. Rivista dell'Istituto di Storia dell'Europa mediterranea, 6, pp. 189-222. 